\title{
Future aspects of halloysite nanotubes in forensic investigations
}

\begin{abstract}
Nanotechnology-based materials have started gaining attention in the field of Forensic Science during investigations. In future, the nanomaterials can enable easy collection and along with rapid and specific detection of evidence from crime scenes. This article focused on the aspects of Halloysite Nanotubes, a clay-based nanomaterial, in forensic investigations. They have the potential to be used in the development of fingerprints as well as detection of evidence such as biological agents, explosives and toxic gases during forensic investigations.
\end{abstract}

Keywords: Forensic science, Halloysite nanotubes, Nanotechnology, Latent fingerprint
Volume 6 Issue 2 - 2017

Gaurav Pandey, Deepak Rawtani, Agrawal YK

Gujarat Forensic Sciences University, India

Correspondence: Deepak Rawtani, Gujarat Forensic Sciences University, Sector I8A, Near Police Bhawan, Gandhinagar, India, Email rawtanid@gmail.com

Received: August 08, 2017 | Published: September 27, 2017

\section{Abbreviations:HNTs, Halloysite Nanotubes; AFM, Atomic Force Microscopy}

\section{Introduction}

Forensic science is a wide field, which comprises of contributions of different branches such as physics, biology, chemistry, geology along with mathematics for the examination and analysis of various evidence collected from a crime scene. In recent times, Nanotechnology has enabled the fast, specific and sensitive analysis of evidence. Nanotechnology-basedtechniques such as Atomic Force Microscopy (AFM) have eased the detection of evidence such as explosives, human hair, line crossings and blood . Various nanomaterials such as silver and gold nanoparticles have also been used for the detection as well as collection of evidence. Among various kinds of evidence, fingerprints have always served as a promising means during forensic investigations. Collection and development of latent fingerprints has always been a problem for investigators. In a literature, bentonite clay has been used for the development of latent fingerprints. The clay was loaded with cationic dyes, rhodamine $6 \mathrm{G}$ and methylene blue, which helped in the development of fingerprints . ${ }^{2}$ Halloysite Nanotubes (HNTs) can also be used for collection and detection of various evidence during forensic investigations.

\section{Halloysite nanotubes: an overview}

HNTs are naturally occurring clay mineral. They are having nanotubular structure with lengths varying from several hundred nanometers to several micrometers . ${ }^{3}$ They have aluminosilicate composition and exhibit positive charge in the inner lumen and negative charge on the outer surface. This type of surface chemistry has enabled their use for loading of various cationic and anionic guest molecules such as enzymes ${ }^{4}$ and dyes.$^{5}$ Their surface can be modified with a variety of polymers to enhance the sensitivity of these materials for various analytes. Non-toxicity and biocompatibility of HNTs have attracted the attention of various researchers around the globe for using them in various biological and pharmaceutical applications . However, in the field of forensic science, the properties of HNTs such as large surface area, tubular lumen, excellent adsorption capacity and tunable surface chemistry can be helpful while collecting evidence such as fingerprints and during detection of samples such as DNA and explosives.

\section{Halloysite nanotubes in forensic investigations In evidence collection}

HNTs exhibit large surface area and their structure resembles a sheet rolled into a cylindrical form. Clay-based materials have been used in past for the collection of latent fingerprints.$^{2}$ HNTs can easily replace such materials due to their large and specific surface area. This feature of HNTs can help in collecting and developing the barely visible latent fingerprints from crime scenes. The nanoscale structure of HNTs can enable their spreading over a large sample collection area and assist in developing and collecting the fingerprints with ease. HNTs have been loaded with various dyes in the past . ${ }^{5}$ This ability can be utilized during fingerprint development by immobilizing fluorescent agents and dyes on the surface and inside the lumen of HNTs.

\section{In evidence detection}

The tunable surface chemistry has facilitated the use of HNTs for the detection of various molecules such as glucose, pesticides, proteins and nucleic acids. This property of HNTs can be exploited in the detection of various evidence collected from crime scenes. Previous studies have shown that gold nanoparticles (AuNPs) upon immobilization in HNTs help in detection of DNA as well as differentiate between single and double stranded DNA. ${ }^{7}$ This ability of HNTs in combination with AuNPs can be helpful in detection of single and double stranded DNA samples during forensic investigations. Apart from nucleic acid, surface of HNTs can be tuned accordingly for the detection of various cationic and anionic biological agents used during terrorist activities. The surface chemistry can also play an important role in the detection of various explosives from crime scenes. Excellent adsorption capacity of HNTs can help in the detection of various toxic and explosive gases used in the course of terrorist attacks. In recent times, HNTs have also been explored in the development of nanosensors. Such sensors can also give rapid and on the spot results about the presence of evidence such as explosives, nucleic acids, toxic gases, etc. during forensic investigations.

\section{Conclusion}

Forensic Science has started exploring various types of nanomaterials during forensic investigations for different applications. Halloysite nanotubes have properties such as large surface area, 
tunable surface chemistry and excellent adsorption capacity, which can be explored for forensic investigations. The nanomaterial needs to be explored for different applications in forensic investigations such as development of fingerprints and detection of explosives and gases. Additionally, intensive research needs to be done for exploring the potential of HNTs in the investigation of firearm incidents and soil samples from crime scenes.

\section{Conflicts of interest}

The authors declare no conflict of interest.

\section{Acknowledgments}

There were no funding sources for the study.

\section{References}

1. Pandey G, Tharmavaram M, Rawtani D et al. Multifarious applications of atomic force microscopy in forensic science investigations. Foren $\mathrm{Sci}$ Int. 2017;273:53-63.
2. Chen Q, Kerk W, Soutar A et al. Application of dye intercalated bentonite for developing latent fingerprints. Appl Clay Sci. 2009;44(1-2):156-160.

3. Rawtani D, Agrawal YK Halloysite as support matrices: A review. Emer Mater Res. 2012;1(4):212-220.

4. Pandey G, Munguambe DM, Tharmavaram M et al. Halloysite nanotubes - An efficient 'nano-support' for the immobilization of $\alpha$-amylase. Appl Clay Sci. 2017;136:184-191.

5. Rawtani D, Agrawal YK A Study of the Behavior of HNT with DNA Intercalator Acridine Orange. Bio Nano Sci. 2013;3:52-57.

6. Rawtani D, Agrawal YK Multifarious Applications of Halloysite Nano Tubes: A Review. Rev Adv Mater Sci. 2012;30:282-295.

7. Rawtani D, Agrawal YK Study the interaction of DNA with Halloysite nanotube-gold nanoparticle based composite. J Bio Nano Sci. 2012;6:95-98 